\title{
Characteristics, calibration and measurement uncertainty of RF field strength meters in the frequency range up to $18 \mathrm{GHz}$
}

\author{
R. Pape, K. Münter, and T. Schrader \\ Physikalisch-Technische Bundesanstalt, Bundesallee 100, 38116 Braunschweig, Germany
}

\begin{abstract}
Due to their construction, the devices used for the measurement of electromagnetic fields (radiation monitors) must be calibrated individually. The measurement data obtained are documented in a calibration certificate and the user must take them into account in the uncertainty budget he has to draw up. In addition to the uncertainty contributions of the field representation and of the transfer, other contributions occur which depend on the special constructive features of these devices. When the uncertainty budget is drawn up, it depends on the application and the information about the field structure which contributions will have an effect and which will not. The procedure will be explained by an example.
\end{abstract}

\section{Introduction}

RF field strength meters (radiation monitors) are often used for occupational safety measurements and for tests of electromagnetic compatibility. Their readout value is not only determined by the external field strength, but also depends on some other parameters, e.g. frequency, polarization and modulation. To obtain the best possible results, these characteristics have to be considered during measurements and for the calibration procedure.

\section{Construction of field strength measuring instruments}

Field strength measuring instruments normally consist of a broad-band $E$ or $H$-field sensor with a display unit device and/or a data transmission unit which are interconnected by a cable or a rigid handle. The sensors contain short dipoles or loop antennas which are mostly equipped with diodes or thermocouples as detectors. In practical applications, isotropic

Correspondence to: R. Pape

(reiner.pape@ptb.de) broad-band sensors, whose indication shall be independent of the direction of incidence and polarization of the wave, are in most cases used in view of their easy handling. As this is possible only approximately, the most important contributions to the uncertainty of measurement result from:

- frequency response, anisotropy, non-linearity or limited dynamic range of the sensor,

- temperature dependence of the complete device,

- stability of the amplifier, digitalization error,

- interference of the field by the connecting cable, backscatter from the display unit, influence of auxiliary devices (supports, transmission line for remote reading, etc.).

\section{Calibration procedure}

"Calibration" generally refers to the detection of a deviation between the value indicated by the measuring instrument and the standard value of the physical quantity which must have been derived ("traced back") from the basic quantities of the International System of Units (SI). For field strength measuring instruments this means that a high-frequency field is generated whose strength is well known with the highest possible accuracy and to which the measuring instrument is exposed. The conditions prevailing during calibration must be as close as possible to those of later practical applications so that the above-mentioned contributions to the measurement uncertainty are detected and can be taken into account. With the calibration data the user can calculate from the value indicated by his instrument the best estimate for the field strength for his own measurements and to determine in addition the overall uncertainty of his measurement result. Depending on the case of application, a distinction is first to be made of whether the complete instrument or (in special cases) only

Published by Copernicus Publications on behalf of the URSI Landesausschuss in der Bundesrepublik Deutschland e.V. 


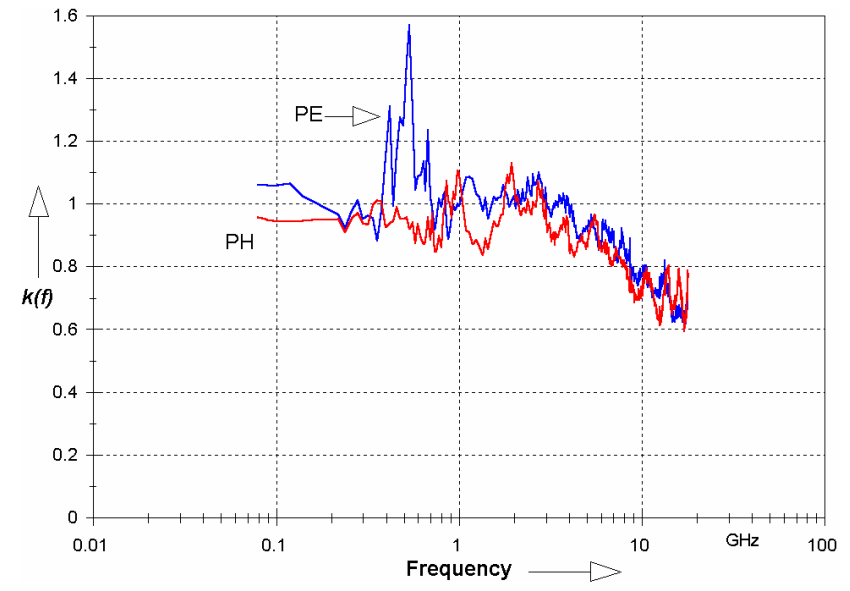

Fig. 1. Frequency response in the " $\boldsymbol{P} \boldsymbol{E}$ " and " $\boldsymbol{P} \boldsymbol{H}$ " orientations.

the sensor is exposed to the field. During measurements for personal protection or in an EMC laboratory usually the complete instrument is exposed. However, this also causes a larger uncertainty, therefore only this unfavourable case will be regarded further on.

During calibration, the complete instrument is placed into the electromagnetic field in defined orthogonal orientations. The mechanical centre of the sensor head is held in a fixed position while the connection to the display unit is consecutively oriented parallel to the electrical $(\boldsymbol{P E})$ field vector, to the magnetic $(\boldsymbol{P} \boldsymbol{H})$ field vector and to the poynting vector $(\boldsymbol{P S})$. Figures 1 and 2 show the impact of the different orientations on the frequency response. The figures illustrate the quotient $k(f)$ ("calibration factor") which is determined during calibration from the incident field strength and the value indicated by the instrument:

$k(f)=E_{\text {Inc }} / E_{\text {Read }}$

The extrema of these three measurement series yield the anisotropy - in the case of this instrument, the calibration factor varies as a function of the frequency by a factor up to 5.

Frequently, the term anisotropy is also used where rotation asymmetry is meant, for example in the IEEE-1309 standard. Such a measurement, in which the instrument is rotated only about its fixed longitudinal axis shows the different sensitivities of the antenna elements and it is not more than a functional test.

In a special orientation $(\boldsymbol{P} \boldsymbol{S})$, backscatter of the housing also leads to a large anisotropy which can, however, be reduced by absorber material (Fig. 3).

The measured anisotropy data for diode detectors are valid only in a limited dynamic range in which their characteristic curve can be regarded as quadratic. In the case of larger field strengths they become linear, and thus correct vectorial addition of the signals of the three single antennas is no longer possible. This dynamic limitation must also be taken into

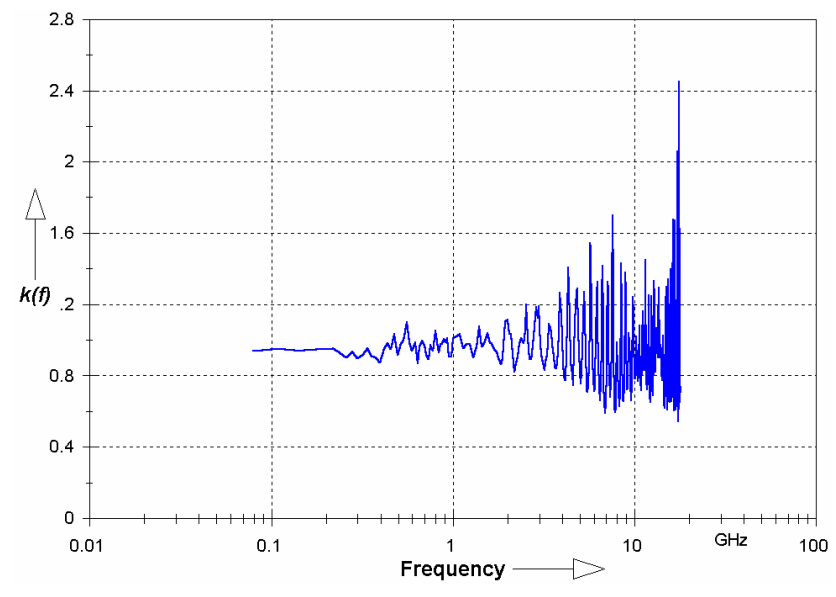

Fig. 2. Frequency response in the "PS" orientation.

account by a correction factor $k(\bmod )$ for measurements in amplitude-modulated fields, as is shown in Fig. 4. Up to approx. $50 \mathrm{~V} / \mathrm{m}$, the probe investigated works as an r.m.s. value rectifier - i.e. in the quadratic part of the characteristic line. Higher field strengths cause peak rectification in the linear part of the characteristic curve, thus the correction factor dependends on the modulation degree and field strength as well.

\section{Measurement uncertainty}

In practical applications it is often advisable to combine the results of the calibration to one overall correction factor which the user can apply directly to convert the display value $\left(E_{\text {Read }}\right)$ of his instrument into the best estimate $\left(E_{\text {Meas }}\right)$ for the unknown field strength. The model equation for this process is simply:

$E_{\text {Meas }}=k(f, I s o, T, \operatorname{lin} \ldots) E_{\text {Read }}$

If we assume furthermore that the indicated value of the instrument (at least in case of a high-resolution digital display) does not show any relevant uncertainty in this application, then the overall uncertainty for the field strength $E_{\text {Meas }}$ results from the uncertainty of the correction factor alone!

However, this simple approach presupposes that it will be possible to combine the influences so far discussed and the single correction factors resulting therefrom in a technically suitable model equation to obtain an overall correction factor $k(f, I s o ., T, \operatorname{lin} \ldots)$ which is valid for the special instrument under the current conditions of use. If the single correction factors are assumed to be independent and uncorrelated, and without additional informations, it is obvious that their product is a good assumption for the overall correction factor. This leads to the general expression:

$k(f, I$ so. $, T, \operatorname{lin} \ldots)=k(f) \quad k(\operatorname{Iso}) k(T) \quad k(\operatorname{lin}) \ldots$

In this case it is possible to determine the single correction factors by separate calibration measurements for the 


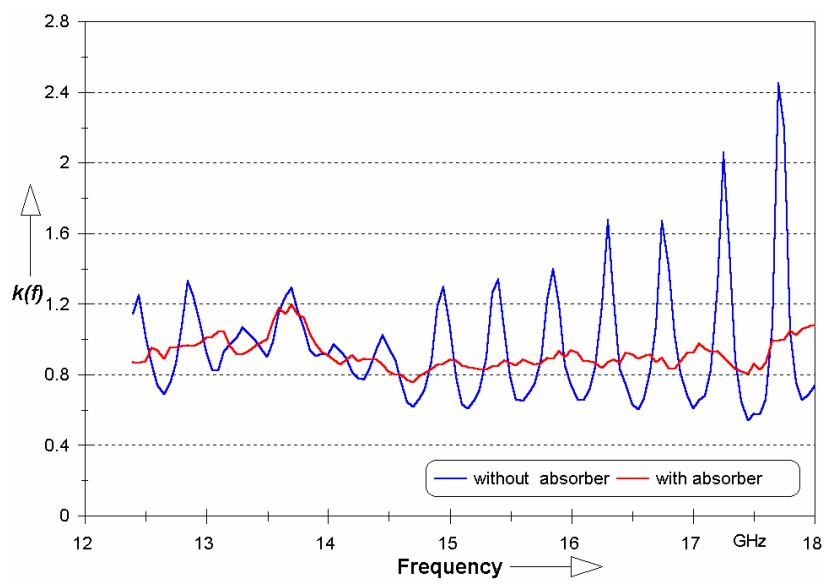

Fig. 3. Reflection of the housing and its damping for "PS" orientation.

respective measuring instrument, and the respective uncertainty contributions will be obtained. If this is not feasible in the individual case, reliable well-founded empirical values, data from literature or manufacturer information must alternatively be referred to.

In an uncertainty budget based on the above equation which - if possible - should be generally valid, at first all correction factors to be expected are provided so that in the special case of application only the current numerical values must be entered to calculate the overall uncertainty. If the user considers single correction factors to be irrelevant, he sets their numerical value to 1 and the uncertainty to 0 , to make it ineffective in the overall budget. The procedure can best be explained by an example:

The measurement task is the following: a technician has to control the personal protection limiting value during work $(L V)$ and must adjust the alarm threshold of his radiation monitor accordingly. The specifications are:

Measurement on a D-network base station in the upper band between approx. $935 \mathrm{MHz}$ and $960 \mathrm{MHz}$, personal protection limiting value $L V=42 \mathrm{~V} / \mathrm{m}$.

The calibration certificate furnishes three frequency responses according to the three orientations measured during calibration. These frequency responses contain the correction factors the technician can use to convert the readout of his instrument - at an electrical field strength of $40 \mathrm{~V} / \mathrm{m}-$ into the best possible estimate for the field strength, if he knows the frequency and the polarization of the field. For the single frequency responses, an expanded relative uncertainty of $1.2 \mathrm{~dB}$ (approx. 12\%) is stated. These data furnish the extremes $k(f)_{M I N}=0.79$ and $k(f)_{M A X}=1.08$ in the required frequency range. The interval of the correction factor which considers both the anisotropy and the frequency response of his radiation monitor is thus limited even if the polarization of the field is completely unknown. Without additional informations, he assumes a rectangular distribution

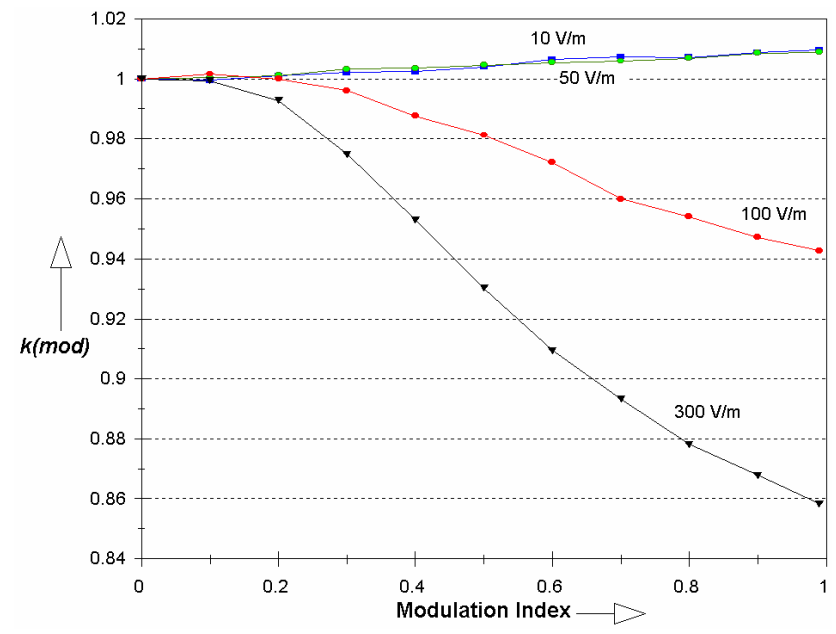

Fig. 4. Behaviour at amplitude modulation.

and regards the mean value $\left(k(f)_{C A L}=0.935\right)$ according to Eq. (4) of this interval as the best estimate for the correction factor valid for the whole D-network frequency range. Both interval limits have the uncertainty stated in the calibration certificate for the different frequency responses, which are fully correlated. Introducing $k(f)_{C A L}$ with that stated uncertainty therefore compensates the frequency response in the best possible way, even when the polarization is completely unknown. In this case a readout of $D V=44.9 \mathrm{~V} / \mathrm{m}$ on the instrument corresponds to the limiting value from Eq. (5). Dividing the interval limits by $k(f)_{C A L}$ normalizes the mean value to 1 , so $k(f)_{I S O}=1$ is introduced as a fictive anisotropy correction factor with no uncertainty coming from the frequency response calibration, because $k(f)_{C A L}$ already takes that into account. Therefore $k(f)_{\text {ISO }}$ does not cause a numerical change, and its associated uncertainty is caused by the anisotropic sensitivity. The additional variance is calculated from the normalized interval limits, assuming a rectangular distribution as mentioned above. With this concept the uncertainty contributions are well separated - the contribution of the laboratory producing the calibration field is in $k(f)_{C A L}$, and the anisotropic instrument response is in $k(f)_{\text {ISO }}$.

As the temperature dependence of the radiation monitor has not been investigated during the calibration, the technician introduces an additional correction factor $k(T)$ for it, with a numerical value of 1 as the best estimate and the relative uncertainty of $5 \%$ stated by the manufacturer over the temperature range required here. For values from the literature without detailed specification, a rectangular distribution for this parameter is appropriate. Now he has inserted all available informations into the uncertainty budget, which finally takes the following form (e.g. calculated with a special computer program, here "GUM Workbench"). 
Table 1. Uncertainty budget.

\begin{tabular}{llllll}
\hline Quantity & Value & Standard uncertainty & Degree of freedom & Uncertainty contribution & Index \\
\hline$k(f)_{M I N}$ & 0.7900 & 0.0474 & 50 & $-1.1 \mathrm{~V} / \mathrm{m}$ & $5.9 \%$ \\
$k(f)_{M A X}$ & 1.0800 & 0.0650 & 50 & $-1.6 \mathrm{~V} / \mathrm{m}$ & $11.2 \%$ \\
$k(f)_{C A L}$ & 0.9350 & 0.0402 & & & \\
$k(f)_{I S O}$ & 1.0000 & 0.0895 & $\infty$ & $-4.1 \mathrm{~V} / \mathrm{m}$ & $75.2 \%$ \\
$k(T)$ & 1.0000 & 0.0289 & $\infty$ & $-1.3 \mathrm{~V} / \mathrm{m}$ & $7.7 \%$ \\
$L V$ & $42.0 \mathrm{~V} / \mathrm{m}$ & & & & \\
$D V$ & $44.9 \mathrm{~V} / \mathrm{m}$ & $5.04 \mathrm{~V} / \mathrm{m}$ & 1700 & & \\
\hline
\end{tabular}

4.1 List of quantities and values

$k(f)_{M I N}: \quad$ Calibration data (minimum) (type B standard distribution)

value: 0.79 expanded uncertainty: 0.0948 coverage factor: 2

$k(f)_{M A X}: \quad$ Calibration data (maximum) (type B standard distribution)

value: 1.08 expanded uncertainty: 0.13 coverage factor: 2

$k(f)_{C A L}: \quad$ Correction factor $=$ mean value of the interval (intermediate result)

$k(f)_{I S O}: \quad$ Correction factor for norm. anisotropy interval (type $\mathrm{B}$ rectangular distribution)

value: 1 half width of the limits: 0.155

$k(T)$ : $\quad$ Correction factor for temperature variation (type $\mathrm{B}$ rectangular distribution)

value: 1 half width of the limits: 0.05

$L V: \quad$ Empty field strength, here: limiting value specification acc. to 26. BImSchV (constant) value: $42 \mathrm{~V} / \mathrm{m}$

$D V: \quad$ Display value of this instrument for field strength limiting value (result)

\subsubsection{Model equations}

$k(f)_{C A L}=\left(k(f)_{M I N}+k(f)_{M A X}\right) / 2$

$D V=L V /\left(k(f)_{C A L} k(f)_{I S O} k(T)\right)$

Correlation coefficients: $r\left(k(f)_{M I N}, k(f)_{M A X}\right)=1$.

Table 1 shows the complete uncertainty budget and furnishes as result:

quantity: $D V=45 \mathrm{~V} / \mathrm{m} \quad$ expanded uncertainty: $\pm 10 \mathrm{~V} / \mathrm{m}$

coverage factor: 2.0 coverage: $t$-table 95 .
In this example, the deviation of the corrected readout from the limiting value is only relatively small. However, if the technician would simply set the alarm threshold of his instrument to $45 \mathrm{~V} / \mathrm{m}$, the personal protection limiting value would already be exceeded with a probability of $50 \%$ ! Therefore, to remain with a probability of more than $95 \%$ below the limiting value when the earliest warning signal is given, he sets the threshold lower by the expanded uncertainty of $10 \mathrm{~V} / \mathrm{m}$. Setting the alarm threshold to a display value of $35 \mathrm{~V} / \mathrm{m}$ should therefore meet his safety requirements even under his unfavourable measurement conditions.

\section{Conclusions}

The result of this example clearly shows that the anisotropy makes the dominating contribution to the overall uncertainty. This fact retrospectively justifies the extensive work which was necessary during calibration, but also the considerations of the user himself. It also shows that already during calibration of such devices, the later field of application should be taken into consideration. From the viewpoint of the user it would be desirable if the manufacturers placed devices with a considerably smaller anisotropy on the market.

\section{References}

IEEE Std 1309-1996: IEEE Standard for Calibration of Electromagnetic Field Sensors and Probes, Excluding Antennas, from $9 \mathrm{kHz}$ to $40 \mathrm{GHz}$.

VDI/VDE/DGQ DKD 2622 (sheet 10): Calibration of measuring devices for electrical quantities, high-frequency field strength measuring instruments.

VDI/VDE/DGQ DKD 2622 (sheet 2): Calibration of measuring devices for electrical quantities, determination of the uncertainty of measurement.

"GUM Workbench" Program, description, see http://www. metrodata.de. 